\title{
Nonnegative Scalar Curvature and Area Decreasing Maps
}

Weiping ZHANG

Chern Institute of Mathematics 85 LPMC, Nankai University, Tianjin 300071, P.R. China E-mail: weiping@nankai.edu.cn

URL: http://old.cim.nankai.edu.cn/members/user/weiping/?name=weiping

Received December 18, 2019, in final form April 15, 2020; Published online April 22, 2020

https://doi.org/10.3842/SIGMA.2020.033

Abstract. Let $\left(M, g^{T M}\right)$ be a noncompact complete spin Riemannian manifold of even dimension $n$, with $k^{T M}$ denote the associated scalar curvature. Let $f: M \rightarrow S^{n}(1)$ be a smooth area decreasing map, which is locally constant near infinity and of nonzero degree. We show that if $k^{T M} \geq n(n-1)$ on the support of $\mathrm{d} f$, then $\inf \left(k^{T M}\right)<0$. This answers a question of Gromov. We use a simple deformation of the Dirac operator to prove the result. The odd dimensional analogue is also presented.

Key words: scalar curvature; spin manifold; area decreasing map

2020 Mathematics Subject Classification: 53C27; 57R20; 58J20

\section{Introduction}

It is well-known that starting with the famous Lichnerowicz vanishing theorem [5], Dirac operators have played important roles in the study of Riemannian metrics of positive scalar curvature on spin manifolds (cf. [3] and [4]). A notable example is Llarull's rigidity theorem [6] which states that for a compact spin Riemannian manifold $\left(M, g^{T M}\right)$ of dimension $n$ such that the associated scalar curvature $k^{T M}$ verifies that $k^{T M} \geq n(n-1)$, any (non-strict) area decreasing smooth map $f: M \rightarrow S^{n}(1)$ of nonzero degree is an isometry.

Recently, Gromov states in [2, p. 45] a noncompact extension of the Llarull theorem. Namely, if $\left(M, g^{T M}\right)$ is an $n$ dimensional noncompact complete spin Riemannian manifold, $f: M \rightarrow S^{n}(1)$ a smooth (non-strict) area decreasing map (which is locally constant near infinity) of nonzero degree such that $k^{T M} \geq n(n-1)$ on the support of $\mathrm{d} f$, then

$$
\inf \left(k^{T M}\right) \leq 0
$$

The argument used by Gromov for (1.1) relies on the relative index theorem of GromovLawson [3], which depends on the positivity of $k^{T M}$ near infinity. Gromov then raises the question that whether the inequality in (1.1) can actually be made strict.

The purpose of this short note is to provide a positive answer to this question when $n$ is even. That is, (1.1) can indeed be improved to $\inf \left(k^{T M}\right)<0$. When $n$ is odd, we improve (1.1) to $\inf \left(k^{T M}\right)<0$ under the condition that $k^{T M}>n(n-1)$ on the support of $\mathrm{d} f$.

The main idea of the proof, similar to [8, equation (1.11)], is to deform the involved twisted Dirac operator (constructed as in [6]) on $M$ by a suitable endomorphism of the twisted vector bundle (cf. (2.8)). The deformed Dirac operator turns out to be invertible near infinity, and one can then apply the relative index theorem to complete the proof.

This paper is a contribution to the Special Issue on Scalar and Ricci Curvature in honor of Misha Gromov on his 75th Birthday. The full collection is available at https://www.emis.de/journals/SIGMA/Gromov.html 


\section{The main results and their proofs}

This section is organized as follows. In Section 2.1, we restate the main results of this paper. In Sections 2.2 and 2.3, we prove the main results stated in Section 2.1.

\subsection{The main results}

Let $\left(M, g^{T M}\right)$ be an $n$-dimensional noncompact spin complete Riemannian manifold. Let $k^{T M}$ be the associated scalar curvature. Let $S^{n}(1)$ be the standard $n$-dimensional unit sphere carrying its canonical metric. Following [3], a smooth map $f: M \rightarrow S^{n}(1)$ is called area decreasing if for any two form $\alpha \in \Omega^{2}\left(S^{n}(1)\right), f^{*} \alpha \in \Omega^{2}(M)$ verifies that

$$
\left|f^{*} \alpha\right| \leq|\alpha|
$$

We now assume that $f: M \rightarrow S^{n}(1)$ is a smooth area decreasing map such that it is locally constant near infinity. That is, it is locally constant outside a compact subset $K \subset M$. We also assume that

$$
\operatorname{deg}(f) \neq 0
$$

Let $\mathrm{d} f: T M \rightarrow T S^{n}(1)$ be the differential of $f$. The support of $\mathrm{d} f$ is defined to be $\operatorname{Supp}(\mathrm{d} f)=$ $\overline{\{x \in M: \mathrm{d} f(x) \neq 0\}}$.

The main result of this short note can be stated as follows.

Theorem 2.1. Under the above assumptions, if $n$ is even and

$$
k^{T M} \geq n(n-1) \quad \text { on } \quad \operatorname{Supp}(\mathrm{d} f),
$$

then one has

$$
\inf \left(k^{T M}\right)<0 .
$$

When $n$ is odd, we have the following analogue which shows that (2.4) still holds when the inequality (2.3) holds strictly.

Theorem 2.2. Under the assumptions above Theorem 2.1, if $n$ is odd and

$$
k^{T M}>n(n-1) \quad \text { on } \operatorname{Supp}(\mathrm{d} f),
$$

then (2.4) still holds.

Theorems 2.1 and 2.2 will be proved in Sections 2.2 and 2.3 respectively.

\subsection{Proof of Theorem 2.1}

Let $S(T M)=S_{+}(T M) \oplus S_{-}(T M)$ be the $\mathbf{Z}_{2}$-graded Hermitian vector bundle of spinors associated to $\left(T M, g^{T M}\right)$, carrying the canonically induced Hermitian connection $\nabla^{S(T M)}=$ $\nabla^{S_{+}(T M)}+\nabla^{S_{-}(T M)}$ (cf. [4]). And we use similar notation for $S^{n}(1)$.

Following [6], let $E=E_{+} \oplus E_{-}$be the $\mathbf{Z}_{2}$-graded Hermitian vector bundle

$$
f^{*}\left(S\left(T S^{n}(1)\right)\right)=f^{*}\left(S_{+}\left(T S^{n}(1)\right)\right) \oplus f^{*}\left(S_{-}\left(T S^{n}(1)\right)\right)
$$

over $M$ carrying the pull-back Hermitian connection $\nabla^{E}=\nabla^{E_{+}}+\nabla^{E_{-}}$. Let $R^{E}=\left(\nabla^{E}\right)^{2}$ be the curvature of $\nabla^{E}$. 
Let $D^{E}: \Gamma(S(T M) \widehat{\otimes} E) \rightarrow \Gamma(S(T M) \widehat{\otimes} E)$ be the canonically defined (twisted by $E$ ) Dirac operator (cf. [4]). ${ }^{1}$ Then one has the canonical splitting $D=D_{+}+D_{-}$with $D_{+}^{E}: \Gamma\left(S_{+}(T M) \otimes\right.$ $\left.E_{+} \oplus S_{-}(T M) \otimes E_{-}\right) \rightarrow \Gamma\left(S_{-}(T M) \otimes E_{+} \oplus S_{+}(T M) \otimes E_{-}\right)$, while $D_{-}^{E}: \Gamma\left(S_{+}(T M) \otimes E_{-} \oplus\right.$ $\left.S_{-}(T M) \otimes E_{+}\right) \rightarrow \Gamma\left(S_{-}(T M) \otimes E_{-} \oplus S_{+}(T M) \otimes E_{+}\right)$. Moreover, one has formally that

$$
\left(D_{+}^{E}\right)^{*}=D_{-}^{E} .
$$

Take any $p \in S^{n}(1) \backslash f(M \backslash K)$. Let $X \in T S^{n}(1)$ be a smooth vector field on $S^{n}(1)$ such that $|X|>0$ on $S^{n}(1) \backslash\{p\}$. Let $v=c(X): S_{+}\left(T S^{n}(1)\right) \rightarrow S_{-}\left(T S^{n}(1)\right)$ be the Clifford action of $X$. Let $v^{*}: S_{-}\left(T S^{n}(1)\right) \rightarrow S_{+}\left(T S^{n}(1)\right)$ be the adjoint of $v$ with respect to the Hermitian metrics on $S_{ \pm}\left(T S^{n}(1)\right)$. Let $V: S\left(T S^{n}(1)\right) \rightarrow S\left(T S^{n}(1)\right)$ be the self-adjoint odd endomorphism defined by

$$
V=v+v^{*} .
$$

Then one has

$$
V^{2}=|X|^{2}
$$

Thus $V$ is invertible on $f(M \backslash K)=\overline{f(M \backslash K)}$. Also, $f^{*} V$ extends to an action on $S(T M) \widehat{\otimes} E$ such that for any $\alpha \in S(T M), u \in E$, one has $\left(f^{*} V\right)(\alpha \widehat{\otimes} u)=(-1)^{\operatorname{deg}(\alpha)} \alpha \widehat{\otimes}\left(f^{*} V\right)(u)$ (cf. [7]).

Let $U_{\frac{1}{2}} \subset M$ be the subset defined by $U_{\frac{1}{2}}=\left\{x \in \operatorname{Supp}(\mathrm{d} f):|\mathrm{d} f(x)|<\frac{1}{2}\right\}$. Let $V_{\frac{1}{2}} \subset M$ be the open subset defined by $V_{\frac{1}{2}}=\left\{x:\left|\wedge^{2}(\mathrm{~d} f(x))\right|>\frac{1}{2}\right\}$, where $\wedge^{2}(\mathrm{~d} f)$ is the induced action of $\mathrm{d} f$ on the exterior product $\wedge^{2}(T M)$. Clearly, $\bar{U}_{\frac{1}{2}} \cap \bar{V}_{\frac{1}{2}}=\varnothing$.

Let $\varphi: M \rightarrow[0,1]$ be a smooth function such that $\varphi=1$ on $(M \backslash \operatorname{Supp}(\mathrm{d} f)) \cup U_{\frac{1}{2}}$, while $\varphi=0$ on $V_{\frac{1}{2}}$. The existence of $\varphi$ is clear.

Similar to [8, equation (1.11)], for any $\varepsilon>0$, let $D_{\varepsilon}^{E}: \Gamma(S(T M) \widehat{\otimes} E) \rightarrow \Gamma(S(T M) \widehat{\otimes} E)$ be the deformed twisted Dirac operator defined by ${ }^{2}$

$$
D_{\varepsilon}^{E}=D^{E}+\varepsilon \varphi f^{*} V .
$$

Let $D_{\varepsilon,+}^{E}: \Gamma\left(S_{+}(T M) \otimes E_{+} \oplus S_{-}(T M) \otimes E_{-}\right) \rightarrow \Gamma\left(S_{-}(T M) \otimes E_{+} \oplus S_{+}(T M) \otimes E_{-}\right)$and $D_{\varepsilon,-}^{E}: \Gamma\left(S_{+}(T M) \otimes E_{-} \oplus S_{-}(T M) \otimes E_{+}\right) \rightarrow \Gamma\left(S_{-}(T M) \otimes E_{-} \oplus S_{+}(T M) \otimes E_{+}\right)$be the natural restrictions.

From (2.8), one has

$$
\left(D_{\varepsilon}^{E}\right)^{2}=\left(D^{E}\right)^{2}+\varepsilon c(\mathrm{~d} \varphi) f^{*} V+\varepsilon \varphi\left[D^{E}, f^{*} V\right]+\varepsilon^{2} \varphi^{2} f^{*}\left(V^{2}\right),
$$

where $c(\cdot)$ is the notation for the Clifford action, $[\cdot, \cdot]$ is the notation for the supercommutator in the sense of [7], and we identify $\mathrm{d} \varphi$ with the gradient of $\varphi$.

Let $e_{1}, \ldots, e_{n}$ be an orthonormal basis of $\left(T M, g^{T M}\right)$. By the definition of the Dirac operator, $D^{E}=\sum_{i=1}^{n} c\left(e_{i}\right) \nabla_{e_{i}}$, one has (cf. [8])

$$
\left[D^{E}, f^{*} V\right]=\sum_{i=1}^{n} c\left(e_{i}\right) f^{*}\left(\nabla_{f_{*}\left(e_{i}\right)}^{S\left(T S^{n}(1)\right)} V\right) .
$$

Since by definition $\varphi=1$ on $M \backslash K$, while $f$ is locally constant on $M \backslash K$, from (2.9) and (2.10) we see that the following identity holds on $M \backslash K$,

$$
\left(D_{\varepsilon}^{E}\right)^{2}=\left(D^{E}\right)^{2}+\varepsilon^{2} f^{*}\left(V^{2}\right) .
$$

\footnotetext{
${ }^{1}$ Here " $\widehat{\otimes}$ " is the notation for the $\mathbf{Z}_{2}$-graded tensor product (cf. [7]).

${ }^{2}$ In view of [7], one may regard $D_{\varepsilon}^{E}$ as a "super" Dirac operator.
} 
As $\left.V\right|_{f(M \backslash K)}$ is invertible, from (2.11), one sees that there is a constant $a>0$ such that for any $s \in \Gamma(S(T M) \widehat{\otimes} E)$ supported in a compact subset of $M \backslash K$, one has

$$
\left\|D_{\varepsilon}^{E} s\right\| \geq \varepsilon a\|s\| \text {. }
$$

From $(2.12)$, one sees that one can apply the relative index theorem in [3] to $D_{\varepsilon,+}^{E}$ (compare with $\left[1\right.$, Section $\left.\left.6 \frac{4}{5}\right]\right)$. In particular, one gets, by similar computations as in [3] and [4, Proposition III.11.24],

$$
\text { ind } \begin{aligned}
\left(D_{\varepsilon,+}^{E}\right) & =\operatorname{deg}(f)\left\langle\operatorname{ch}\left(S_{+}\left(T S^{n}(1)\right)\right)-\operatorname{ch}\left(S_{-}\left(T S^{n}(1)\right)\right),\left[S^{n}(1)\right]\right\rangle \\
& =(-1)^{\frac{n}{2}} \operatorname{deg}(f) \chi\left(S^{n}(1)\right)=2(-1)^{\frac{n}{2}} \operatorname{deg}(f) .
\end{aligned}
$$

By the Lichnerowicz formula [5] for $D^{E}$ (cf. [4]), one has

$$
\left(D^{E}\right)^{2}=-\Delta^{E}+\frac{k^{T M}}{4}+\frac{1}{2} \sum_{i, j=1}^{n} c\left(e_{i}\right) c\left(e_{j}\right) R^{E}\left(e_{i}, e_{j}\right),
$$

where $-\Delta^{E} \geq 0$ is the corresponding Bochner Laplacian.

From [6, equation (4.6)], one knows that

$$
\frac{1}{2} \sum_{i, j=1}^{n} c\left(e_{i}\right) c\left(e_{j}\right) R^{E}\left(e_{i}, e_{j}\right) \geq-\frac{n(n-1)}{4}\left|\wedge^{2}(\mathrm{~d} f)\right| .
$$

From $(2.1),(2.3),(2.9),(2.14)$ and $(2.15)$, one has that near any $x \in V_{\frac{1}{2}}$,

$$
\left(D_{\varepsilon}^{E}\right)^{2}+\Delta^{E} \geq 0 \text {. }
$$

Near any $x \in \operatorname{Supp}(\mathrm{d} f) \backslash \bar{V}_{\frac{1}{2}}$, by (2.3), (2.9), (2.14) and (2.15), one has

$$
\left(D_{\varepsilon}^{E}\right)^{2}+\Delta^{E} \geq \frac{n(n-1)}{8}+\varepsilon c(\mathrm{~d} \varphi) V+\varepsilon \varphi\left[D^{E}, f^{*} V\right]+\varepsilon^{2} \varphi^{2} f^{*}\left(V^{2}\right) .
$$

From (2.9), (2.10), (2.14) and (2.15), one has that near any $x \in M \backslash \operatorname{Supp}(\mathrm{d} f)$,

$$
\left(D_{\varepsilon}^{E}\right)^{2}+\Delta^{E} \geq \frac{k^{T M}}{4}+\varepsilon^{2} f^{*}\left(V^{2}\right) .
$$

Now assume that (2.4) does not hold. Then one has over $M$ that

$$
k^{T M} \geq 0 .
$$

From $(2.10),(2.11),(2.16)-(2.19)$ and the compactness of $\operatorname{Supp}(\mathrm{d} f)$, we see that when $\varepsilon>0$ is small enough, there is a smooth nonnegative endormorphism $a_{\varepsilon}$ of $S(T M) \widehat{\otimes} E$ such that

$$
a_{\varepsilon}>0 \quad \text { on } \quad(M \backslash K) \cup U_{\frac{1}{2}}
$$

and that one has on $M$ that

$$
\left(D_{\varepsilon}^{E}\right)^{2} \geq-\Delta^{E}+a_{\varepsilon} .
$$

From (2.20) and (2.21), one finds that the relative index of $D_{\varepsilon,+}^{E}$ verifies that

$$
\text { ind }\left(D_{\varepsilon,+}^{E}\right)=0 \text {, }
$$

which contradicts (2.2) and (2.13). This completes the proof of Theorem 2.1.

Remark 2.3. In view of $(2.12)$ and [1, Section $\left.6 \frac{4}{5}\right]$, one sees that the above proof fits with Gromov's suggestion in [2, p. 45] that one may use the Callias type index arguments to deal with (2.4). Also, from the above proof one sees that (2.3) can be weakened to

$$
k^{T M} \geq n(n-1)\left|\wedge^{2}(\mathrm{~d} f)\right| \quad \text { on } \operatorname{Supp}(\mathrm{d} f),
$$

with the inequality being strict on $\operatorname{Supp}(\mathrm{d} f) \backslash\{x \in M: \mathrm{d} f(x) \neq 0\}$. With (2.22) the condition (2.1) is no longer needed. 


\subsection{Proof of Theorem 2.2}

In view of Remark 2.3, we will state and prove the following refined version of Theorem 2.2.

Theorem 2.4. Let $\left(M, g^{T M}\right)$ be a noncompact spin complete Riemannian manifold of odd dimension $n$, and $f: M \rightarrow S^{n}(1)$ be a smooth map which is locally constant near infinity and of nonzero degree. If we assume that the scalar curvature $k^{T M}$ of $g^{T M}$ verifies that

$$
k^{T M}>n(n-1)\left|\wedge^{2}(\mathrm{~d} f)\right| \quad \text { on } \operatorname{Supp}(\mathrm{d} f),
$$

then one has

$$
\inf \left(k^{T M}\right)<0 .
$$

Proof. For any $R>0$, let $S^{1}(R)$ be the round circle of radius $R$, with the canonical metric $\mathrm{d} t^{2}$. Let $M \times S^{1}(R)$ be the complete Riemannian manifold of the product metric $g^{T M} \oplus \mathrm{d} t^{2}$. Following [6], we consider the chain of maps

$$
M \times S^{1}(R) \stackrel{f \times \frac{1}{R}}{\longrightarrow} S^{n}(1) \times S^{1}(1) \stackrel{h}{\rightarrow} S^{n+1}(1),
$$

where $\frac{1}{R}: S^{1}(R) \rightarrow S^{1}(1)$ is the standard shrinking map, and $h$ is a suspension map of degree one such that $|\mathrm{d} h| \leq 1$. Let $f_{R}=h \circ\left(f \times \frac{1}{R}\right): M \times S^{1}(R) \rightarrow S^{n+1}(1)$ denote the composition. Then one has ${ }^{3}$

$$
\operatorname{deg}\left(f_{R}\right)=\operatorname{deg}(f) \neq 0 .
$$

Let $p \in S^{n+1}(1)$ be any regular value of $f_{R}$. Let $X \in T S^{n+1}(1)$ be a smooth vector field on $S^{n+1}(1)$ such that $|X|>0$ on $S^{n+1}(1) \backslash\{p\}$. Let $v=c(X): S_{+}\left(T S^{n+1}(1)\right) \rightarrow S_{-}\left(T S^{n+1}(1)\right)$ be the Clifford action of $X$. Let $V: S\left(T S^{n+1}(1)\right) \rightarrow S\left(T S^{n+1}(1)\right)$ be defined as in (2.6). Then (2.7) holds and $V$ is invertible on $S^{n+1}(1) \backslash\{p\}$. In particular, there is $\delta>0$ such that

$$
V^{2} \geq \delta \quad \text { on } \overline{f_{R}\left((M \backslash \operatorname{Supp}(\mathrm{d} f)) \times S^{1}(R)\right)} .
$$

For simplicity, denote $M_{R}=M \times S^{1}(R)$. Let $E_{R}=f_{R}^{*}\left(S\left(T S^{n+1}(1)\right)\right)$ be the $\mathbf{Z}_{2}$-graded Hermitian vector bundle over $M_{R}$ as in (2.5). Let $D^{E_{R}}: \Gamma\left(S\left(T M_{R}\right) \widehat{\otimes} E_{R}\right) \rightarrow \Gamma\left(S\left(T M_{R}\right) \widehat{\otimes} E_{R}\right)$ denote the canonical twisted Dirac operator.

As in (2.8), for any $\varepsilon>0$, let $D_{\varepsilon}^{E_{R}}: \Gamma\left(S\left(T M_{R}\right) \widehat{\otimes} E_{R}\right) \rightarrow \Gamma\left(S\left(T M_{R}\right) \widehat{\otimes} E_{R}\right)$ be the deformed twisted Dirac operator defined by ${ }^{4}$

$$
D_{\varepsilon}^{E_{R}}=D^{E_{R}}+\varepsilon f_{R}^{*} V .
$$

Let $D_{\varepsilon,+}^{E_{R}}: \Gamma\left(S_{+}\left(T M_{R}\right) \otimes E_{R,+} \oplus S_{-}\left(T M_{R}\right) \otimes E_{R,-}\right) \rightarrow \Gamma\left(S_{-}\left(T M_{R}\right) \otimes E_{R,+} \oplus S_{+}\left(T M_{R}\right) \otimes E_{R,-}\right)$ be the natural restriction.

The analogue of (2.9) now takes the form

$$
\left(D_{\varepsilon}^{E_{R}}\right)^{2}=\left(D^{E_{R}}\right)^{2}+\varepsilon\left[D^{E_{R}}, f_{R}^{*} V\right]+\varepsilon^{2} f_{R}^{*}\left(V^{2}\right) .
$$

Let $K$ be a compact subset of $M$ such that $f: M \rightarrow S^{n}(1)$ is locally constant on $M \backslash K$. From (2.10) and the definition of $f_{R}$, one finds on $(M \backslash K) \times S^{1}(R)$ that

$$
\left[D^{E_{R}}, f_{R}^{*} V\right]=O\left(\frac{1}{R}\right)
$$

\footnotetext{
${ }^{3}$ The degree of $f_{R}$ is well-defined as $f_{R}$ has no regular point near infinity.

${ }^{4}$ There is no need here to introduce the function $\varphi$ as in (2.8), as the inequality in (2.23) is strict.
} 
From (2.26), (2.27) and (2.28), one sees that for any $\varepsilon>0$, there exist $R_{0}>0$ and $b>0$ such that when $R \geq R_{0}$, for any $s \in \Gamma\left(S\left(T M_{R}\right) \widehat{\otimes} E_{R}\right)$ supported in a compact subset of $(M \backslash K) \times S^{1}(R)$, one has

$$
\left\|D_{\varepsilon}^{E_{R}} s\right\| \geq \varepsilon b\|s\|
$$

One can then apply the relative index theorem [3] to $D_{\varepsilon,+}^{E_{R}}$ and get as in (2.13) that

$$
\text { ind }\left(D_{\varepsilon,+}^{E_{R}}\right)=2(-1)^{\frac{n+1}{2}} \operatorname{deg}\left(f_{R}\right) \text {. }
$$

Let $e_{1}, \ldots, e_{n+1}$ be an orthonormal basis of $T M_{R}$. The Lichnerowicz formula (2.14) now takes the form

$$
\left(D^{E_{R}}\right)^{2}=-\Delta^{E_{R}}+\frac{\pi_{R}^{*} k^{T M}}{4}+\frac{1}{2} \sum_{i, j=1}^{n+1} c\left(e_{i}\right) c\left(e_{j}\right) R^{E_{R}}\left(e_{i}, e_{j}\right)
$$

where $\pi_{R}: M \times S^{1}(R) \rightarrow M$ denotes the natural projection.

By [6, p. 68], one has at any $(x, y) \in M \times S^{1}(R)$ that

$$
\frac{1}{2} \sum_{i, j=1}^{n+1} c\left(e_{i}\right) c\left(e_{j}\right) R^{E_{R}}\left(e_{i}, e_{j}\right) \geq-\frac{n(n-1)}{4}\left|\wedge^{2}(\mathrm{~d} f(x))\right|+|\mathrm{d} f(x)| O\left(\frac{1}{R}\right) .
$$

From (2.10), (2.23), (2.32) and the compactness of $\operatorname{Supp}(\mathrm{d} f) \subset M$, one sees that there is $\eta>0$ such that when $\varepsilon>0$ is small enough, the following formula holds on $(\operatorname{Supp}(\mathrm{d} f)) \times S^{1}(R)$,

$$
\frac{\pi_{R}^{*} k^{T M}}{4}+\frac{1}{2} \sum_{i, j=1}^{n+1} c\left(e_{i}\right) c\left(e_{j}\right) R^{E_{R}}\left(e_{i}, e_{j}\right)+\varepsilon\left[D^{E_{R}}, f_{R}^{*} V\right] \geq \eta+\left(\pi_{R}^{*}|\mathrm{~d} f|\right) O\left(\frac{1}{R}\right)
$$

Now we observe that (2.28) indeed holds on $(M \backslash \operatorname{Supp}(\mathrm{d} f)) \times S^{1}(R)$.

From $(2.26),(2.27),(2.28)$ and $(2.31)-(2.33)$, one finds that if (2.24) does not hold, then one can first take $\varepsilon>0$ small enough, and then take $R>0$ large enough to get a positive endomorphism $a_{\varepsilon, R}$ of $S\left(T M_{R}\right) \widehat{\otimes} E_{R}$ such that in addition to (2.29), the following formula also holds,

$$
\left(D_{\varepsilon}^{E_{R}}\right)^{2} \geq-\Delta^{E_{R}}+a_{\varepsilon, R}
$$

From (2.34), one gets

$$
\text { ind }\left(D_{\varepsilon,+}^{E_{R}}\right)=0
$$

which contradicts (2.25) and (2.30). This completes the proof of Theorem 2.4.

\section{Acknowledgements}

The author would like to thank the referees for careful readings and very helpful suggestions. This work was partially supported by NNSFC Grant no. 11931007. 


\section{References}

[1] Gromov M., Positive curvature, macroscopic dimension, spectral gaps and higher signatures, in Functional Analysis on the Eve of the 21st Century, Vol. II (New Brunswick, NJ, 1993), Progr. Math., Vol. 132, Birkhäuser Boston, Boston, MA, 1996, 1-213.

[2] Gromov M., Four lectures on scalar curvature, arXiv:1908.10612.

[3] Gromov M., Lawson Jr. H.B., Positive scalar curvature and the Dirac operator on complete Riemannian manifolds, Inst. Hautes Études Sci. Publ. Math. 58 (1983), 83-196.

[4] Lawson Jr. H.B., Michelsohn M.L., Spin geometry, Princeton Mathematical Series, Vol. 38, Princeton University Press, Princeton, NJ, 1989.

[5] Lichnerowicz A., Spineurs harmoniques, C. R. Acad. Sci. Paris 257 (1963), 7-9.

[6] Llarull M., Sharp estimates and the Dirac operator, Math. Ann. 310 (1998), 55-71.

[7] Quillen D., Superconnections and the Chern character, Topology 24 (1985), 89-95.

[8] Zhang W., Positive scalar curvature on foliations: the enlargeability, in Geometric Analysis, in Honor of Gang Tian's 60th Birthday, Progr. Math., Vol. 333, Birkhäuser, Cham, 2020, 537-544, arXiv:1703.04313. 